\title{
Anti-nf-P2X7 Antibody Ointment BIL-010t
}

National Cancer Institute

\section{Source}

National Cancer Institute. Anti-nf-P2X7 Antibody Ointment BIL-010t. NCI Thesaurus.

Code C124647.

An ointment formulation composed of a purified sheep immunog lobulin $\mathrm{G}(\lg \mathrm{G})$ antibody against the non-functional form of the purinergic $\mathrm{P} 2 \mathrm{X} 7$ receptor (nf-P2X7), with potential antineoplastic activity. Upon topical application of the anti-nf-P2X7 antibody ointment BIL-010t, the antibody binds to nf-P2X7 and inhibits its antiapoptotic activity. This may induce apoptosis and inhibit the growth of nf-P2X7-overexpressing cancer cells. P2X7, an ATP-gated cation-selective channel, plays a role in the induction of apoptosis; nf-P2X7, is upregulated in a variety of cancer cell types while not expressed on normal, healthy cells and is unable to form a large transmembrane, apoptotic pore upon exposure to ATP and prevents apoptosis. 